\title{
CONDUCTANCE OF MESOSCOPIC MAGNETIC SYSTEMS
}

\author{
S. KROMPIEWSKI \\ Institute of Molecular Physics, Polish Academy of Sciences \\ Smoluchowskiego 17, 60-179 Poznań, Poland
}

\begin{abstract}
Theoretical studies of electrical conductance of various nanowires are performed within the framework of a semi-realistic $s-d$ tight-binding model. The presented results concern both homogeneous paramagnetic and ferromagnetic systems as well as trilayers composed of 2 magnetic slabs separated by a nonmagnetic spacer. On the one hand, in the case of the homogeneous systems the attention is focused on conductance quantization, which manifests itself when a contact gets open and conduction decays in a spectacular stepwise way. A new approach is developed by assuming that in the last stage of the breaking of the contact between wires there are fewer and fewer, distributed at random, conduction paths passing through the nanowire cross-section. The corresponding conductances are calculated within the quasiballistic regime, using the Kubo formula and a recursion Green function technique. The results for weak ferromagnets (when both majority and minority bands intersect the Fermi surface) are qualitatively different from those for strong ferromagnets (only the minority bands do), which may explain experimental cumulative conductance histograms of $\mathrm{Fe}$ and $\mathrm{Ni}$. On the other hand, giant magnetoresistances of magnetic trilayers are studied for both current-perpendicular-to-plane and current-in-plane geometries. The corresponding magnetoresistances are compared with each other and with the interlayer exchange coupling.
\end{abstract}

PACS numbers: 73.40.Jn, 75.70.-i, 75.70.Pa, 73.23.Ad

\section{Introduction}

With the advent of modern thin-film and nanofabrication techniques, the interest in small magnetic structures has recently rapidly increased. The reason for this is that, on the one hand, small structures of this type, e.g. in a form of quantum dots, quantum nanowires, or quasi 2-dimensional systems, show quite fascinating properties like resonant-tunneling, Coulomb blockade, and conductance quantization or, in the presence of magnetic field, the Aharanov-Bohm effect and the quantum Hall effect. On the other hand, in the case of structures containing magnetic ingredients there appears a new possibility of controlling electric current by switching on and off magnetic field. This way, the electron spin has entered 
into the modern electronics, and starts playing a crucial role. This is in contrast to traditional electronic devices, like silicon transistors, in which only effective charge is of importance and no use is made of its spin. New devices based on "spin electronics" have been already designed (some of them are reviewed in Physics Today special issue: Magneto-electronics, April 1995). A few remarkable examples which may be evoked in this context are: magnetic bipolar transistor (the first all-metal transistor) [1], metal-gate field effect transistor (with a base in a form of a multilayer) [2], magnetoresistive memories [3], and sensors and reading heads of metallic recording devices [4].

\section{Mesoscopic systems}

The simplest definition of mesoscopic systems is that they have dimensions intermediate between microscopic and macroscopic ones, or more precisely, their dimensions are comparable with a characteristic length scale specific to a given problem of interest. As regards electrical properties for instance, the mesoscopic region may be associated with that where ohmic behaviour collapses in view of the fact that the dimensions of a system are no longer greater than each of the following length scales: (i) the de Broglie wavelength of electrons at the Fermi surface, (ii) the mean free path, and (iii) the phase relaxation length. Additionally, (iv) the spin diffusion length, i.e. the average distance covered by a carrier spin before flipping, is also crucial, although it is usually much greater than the other above-mentioned length scales (at least at low temperatures in impurity-free materials).

In the present paper we shall concentrate on just a few selected problems concerning mesoscopic metallic systems in a form of nanowires with ferromagnetic ingredients, leaving aside the vast well-recognized class of semiconductor-based structures. The main points which are to be addressed within this context are conductance quantization and giant magnetoresistance (GMR). The former is of great cognitive importance in view of the up-to-date scientific debate and controversy concerning conductance of the breaking of point contacts between ferromagnets (see below). The latter, most of the devices mentioned above are based on, is studied in an attempt of shedding more light on intercorrelation of the GMR in the current-perpendicular-to-plane (CPP) geometry and in the current-in-plane (CIP) geometry. To avoid any uncontrollable effects, all the calculations are performed by means of an exactly solvable tight-binding model and within the ballistic (quasiballistic) regime in the absence of thermal excitations (phonons and magnons). Moreover, we are also interested in oscillatory phenomena revealed both by the GMR and interlayer exchange coupling as functions of varying sublayer thicknesses. It is well established that oscillatory phenomena have periods determined by calipers (extremal spanning vectors) of the Fermi surface. We shall have a closer. look at this and show that the depending on the geometry of various periods may come into play.

\section{Ballistic transport}

A classical ballistic point contact consists of two semi-infinite electrodes separated by an insulating barrier with a small opening in it. The diameter of the opening must be smaller than the mean free path and much larger than the elec- 
tron wavelength. The region between the electrodes (constriction or neck) may in fact be of finite length, provided it is short enough so that electrons pass ballisticly through it and its conductance is hardly length-dependent.

\subsection{Preliminaries}

For a small voltage difference between the electrodes and at low temperatures the ballistic or Sharvin conductance $[5,6](\Gamma=I / V)$ is:

$$
\Gamma=\frac{1}{2} e^{2} A \sum_{\nu, \sigma} \frac{\mathrm{d} q}{(2 \pi)^{d}}\left|\widehat{n} v_{\nu, \sigma}(q)\right| \delta\left(\epsilon_{\nu, \sigma}(q)-E_{\mathrm{F}}\right),
$$

where $A$ is the cross-section area (normal to the current direction pointed by the unit vector $\hat{n}), E_{\mathrm{F}}$ is the Fermi energy, $v_{\nu, \sigma}(q)$ and $\epsilon_{\nu, \sigma}(q)$ stand for the velocity and the energy for a Bloch state with the wave vector $q$, band index $\nu$ and spin $\sigma$. Replacing the integration over $q$ by the integration over the Fermi surface sheet one arrives at the following remarkably simple expression:

$$
\Gamma=\frac{e^{2}}{h} \frac{A \pi}{(2 \pi)^{d}} \sum_{\nu, \sigma} S_{\nu \sigma}(\widehat{n})
$$

hence, the ballistic conductance is related to the band structure via the projections $S_{\nu \sigma}(\widehat{n})$ of the Fermi surface sheets onto the $\hat{n}$-plane.

For the free electron gas the projections of the two Fermi hemispheres give $S_{\sigma}=2 \pi k_{\mathrm{F}}^{2}$, and Eq. (2) readily leads to the well-known Sharvin conductance (including spin-degeneracy)

$$
\Gamma=\frac{2 e^{2}}{h} \frac{A k_{\mathrm{F}}^{2}}{4 \pi} .
$$

Similarly in two dimensions $\left(d=2, S_{\sigma}=4 k_{\mathrm{F}}\right)$ one finds

$$
\Gamma=\frac{2 e^{2}}{h} \frac{A k_{\mathrm{F}}}{\pi} \text {. }
$$

Incidentally, it is instructive to compare the ballistic conductance with a diffusive regime result obtained by means of the Boltzmann equation. A diagonal component of the conductance tensor, say $z z$, of the latter may be formally obtained from Eq. (1) by making the following replacement:

$$
\left|\widehat{n} v_{\nu, \sigma}(q)\right| \Longrightarrow \tau_{\nu \sigma}\left|\widehat{z} v_{\nu, \sigma}(q)\right|^{2} / L_{z}
$$

with $\tau$ being the relaxation time and $L_{z}-$ the length. The two conductances would be equivalent if the mean-free path $\tau_{\nu \sigma}(q)\left|v_{\nu, \sigma}(q)\right|$ was $q$ - and $\nu$-independent.

\subsection{Conductance}

The simple expression Eq. (1) is only useful if the system is translationally invariant and the energy dispersion is available. If the system is complex it is convenient to work in the real space representation and exploit the Kubo formulae to study transport phenomena. The conductance may be expressed in terms of Green functions $(G F)$ defined by the following recursion relations: 


$$
\begin{aligned}
& G(i, i)=\left[\left(g^{-1}(i)-T G^{\mathrm{L}}(i-1) T-T G^{\mathrm{R}}(i+1) T\right]^{-1},\right. \\
& G^{\mathrm{L}}(i)=\left[g^{-1}(i)-T G^{\mathrm{L}}(i-1) T\right]^{-1}, \\
& G^{\mathrm{R}}(i)=\left[g^{-1}(i)-T G^{\mathrm{R}}(i+1) T\right]^{-1}, \quad G(i, i+1)=G(i, i) T G^{\mathrm{R}}(i+1),
\end{aligned}
$$

where $i$ labels the atomic planes normal to the current direction $(\widehat{z}), T$ is the diagonal matrix of hopping integrals of rank $n_{\text {band }} L_{x} \times L_{y}, g(i)$ is the isolated monolayer GF and $n_{\text {band }}$ is the number of bands. The Green function $G_{i \sigma}^{\mathrm{L}}\left(G_{i \sigma}^{\mathrm{R}}\right)$ applies to a system in which all the atomic planes with indices greater (less) than $i$ are deleted [7]. Finally, the $d c$ conductance $\Gamma_{\sigma}$ is given by

$$
\Gamma_{\sigma}=\frac{4 e^{2}}{h} \operatorname{Tr}\left\{\widetilde{G}_{\sigma}(i-1, i-1) \hat{T} \widetilde{G}_{\sigma}(i, i) \widehat{T}-\widehat{T} \widetilde{G}_{\sigma}(i, i-1) \widehat{T} \tilde{G}_{\sigma}(i, i-1)\right\},
$$

where $\widetilde{G}$ denotes the imaginary part of $G$.

The systems under consideration are assumed to extend in the $\widehat{z}$ direction from $i=1$ up to $L_{z}$, additionally, for conductance calculations they are sandwiched between two semi-infinite ideal lead wires indexed with $-\infty<i<1$ and $L_{z}<i<\infty$. Within the ideal lead wires all the on-site potentials are independent of $i$, so the GF in the second (or third) equation of the equation set (6) does not depend on $i$ either and may be determined numerically.

\section{4. $s-d$ model}

We use a two-band model with intraatomic hybridization, i.e. the simplest approach which includes the so-called Mott scattering mechanism which consists in scattering conduction electrons into $d$ states. The $s-d$ Hamiltonian has been already used to describe magnetic multilayers, and proved to mimic properly certain properties of real systems $(\mathrm{Co} / \mathrm{Cu}$, see [8]). The Hamiltonian reads

$$
H_{\sigma}=\sum_{i, j, \alpha, \sigma} t_{i, j} c_{i \alpha \sigma}^{\dagger} c_{j \alpha \sigma}+\sum_{i, \alpha, \sigma} V_{i \sigma}^{\alpha} c_{i \alpha \sigma}^{\dagger} c_{i \alpha \sigma}+\sum_{i, \alpha, \beta, \sigma(\alpha \neq \beta)} V_{i_{\sigma}}^{\alpha \beta} c_{i \alpha \sigma}^{\dagger} c_{i \alpha \sigma},
$$

where $t_{i, j}, V_{i \sigma}^{\alpha}, V_{i \sigma}^{\alpha \beta}$ are the hopping integral, the on-site potential, and the hybridization, respectively, whereas $i(j), \alpha(\beta)$, and $\sigma$ stand for the lattice site, the band index, and the spin, respectively. In the case of an ideal, infinite homogeneous system the Hamiltonian has the following eigenvalues:

$$
\epsilon(k)^{ \pm}=\frac{1}{2}\left[\epsilon_{s}(k)+\epsilon_{d}(k)\right] \pm \frac{1}{2} \sqrt{\left[\epsilon_{s}(k)-\epsilon_{d}(k)\right]^{2}+4\left(V^{s d}\right)^{2}},
$$

where (for the simple cubic lattice and $\alpha=s, d) \epsilon_{\alpha}(k)=V_{\sigma}^{\alpha}+2 t_{\alpha}\left(\operatorname{cox} k_{x}+\cos k_{y}+\right.$ $\cos k_{z}$ ).

From now on the following set of parameters will be used: $t_{s}=-1, t_{d}=-0.2$, $V^{s d}=1, V_{\sigma}^{s}=0, V_{\sigma}^{d}=-1$ (paramagnetic case), and for the ferromagnetic case $V_{\uparrow}^{d}=-1$ and $V_{\downarrow}^{d}=-0.2$, with $\left|t_{s}\right|$, and the lattice constant regarded as the energy and length units. Density of states of bulk systems for these parameters are depicted in Fig. 1. 


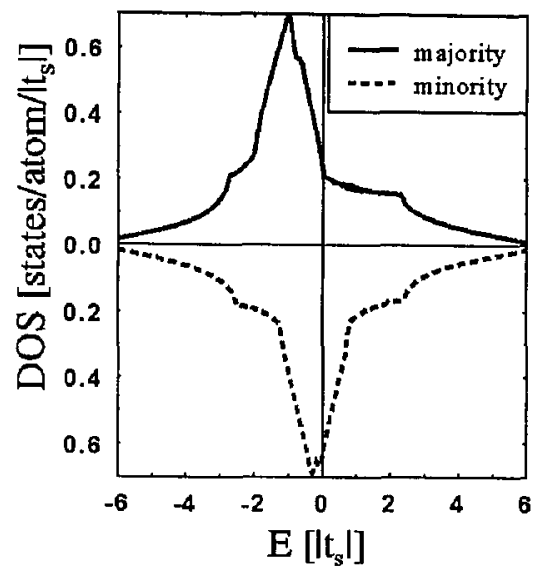

Fig. 1. Majority- and minority-spin electron densities of states of the model bulk ferromagnet. The paramagnetic system is modelled by assuming that the minority band coincides with the majority band.

\section{Conductance quantization}

It is easily seen from Eqs. (3) and (4) that conductance of an ideal ballistic conductor has the following form:

$$
\Gamma=\frac{2 e^{2}}{h} M,
$$

where $e^{2} / h$ is the conductance quantum and $M-$ the total number of allowed conductivity channels determined by the number of transverse modes with energies close to the Fermi surface. This implies that individual conductance steps of $\Gamma$, may be experimentally observable for small constrictions, i.e. well-separated sub-bands. In particular, in two dimensions with the constriction width $L_{y}$, one immediately sees from Eq. (4) that $M=k_{\mathrm{F}} L_{y} / \pi$, i.e. in order for relative changes of $M$ to be substantial, $k_{\mathrm{F}} L_{y}$ must be small. A numerical estimate along this line [9] shows that in the case of the GaAs-AlGaAs heterostructure the constriction must not be thicker than a few hundred $\mathrm{nm}$. In metals the analogous estimate imposes much more rigorous requirements for the constriction width, namely $k_{\mathrm{F}} \sim \AA^{-1}$ implies that in order for $M$ to be a small integer the constriction $A$ has to be of the order of a few $\AA$ only [10].

Conductance quantization (CQ) in ballistic point contacts was first reported in Refs. $[11,12]$ in the two-dimensional gas of a GaAs-AlGaAs heterostructure. The width of the point contacts in those experiments was controlled by applying a (negative) gate voltage. When the absolute value of the gate voltage is large enough, the width of the constriction (in the direction perpendicular to the current) makes the transverse modes quantize and the corresponding sub-bands become well separated from each other [9]. In the case of metallic point contacts it is possible to monitor the stepwise decay of conductance while breaking the circuit, e.g. either by opening an electromagnetic relay or by just bringing two wires to and out of contact [10, 13-15].In this manner one is able to create a constriction (neck) within 
the nanowire of only a few $\AA$ in diameter, which is necessary to get conductance steps visible. So far, a lot of materials have been studied in this way, including: $\mathrm{Au}, \mathrm{Al}, \mathrm{Pb}, \mathrm{Na}, \mathrm{Hg}, \mathrm{Sb}, \mathrm{Co}-\mathrm{Fe}-\mathrm{Si}-\mathrm{B}, \mathrm{Bi}$ and $\mathrm{La}-\mathrm{Sr}-\mathrm{MnO}_{3}$ (see Refs. $[13,14]$ and references therein).

At present there is the consensus that the conductance quantization, in steps of $G_{0}=2 e^{2} / h$, is due to the ballistic transport through a nanocontact between paramagnetic lead wires. In the case of ferromagnetic leads the situation is more complicated and still under debate. On the one hand, experiments on $\mathrm{Ni}$ when performed in air lead to conductance cumulative histograms which are almost featureless $[13,14]$, but are clearly peaked when measured in "vacuum" [16]. On the other hand, for Fe the cumulative histograms have pronounced peaks, according to Ref. [14] even in air. We will show below that these differences may be due to essential differences in electron densities of states between the two transition metals. In fact, bulk $\mathrm{Ni}$ is a strong ferromagnet with its majority-spin $d$-electron band nearly full-filled, whereas $\mathrm{Fe}$, as a weak ferromagnet, has got both the $d$-bands open at the Fermi surface. These two cases may be simulated by the $s-d$ model adopted here, with $E_{\mathrm{F}}$ (Fermi energy) equal to -0.5 and 0 for weak and strong ferromagnets, respectively. The nanocontact in turn is modelled as shown in Fig. 2, i.e. shortly before the contact is open, only a few conduction paths are left and eventually they completely disappear. The conduction paths may, but do not have to, touch one another, they are distributed at random and their length is equal to the contact breaking length (distortion region). It is assumed that there is no correlation between the length and the number of conduction channels. Physics behind this assumption is that: (i) ballistic transport is almost length-independent, and (ii) neither acting forces involved nor surface roughness are actually known. Thus, the present model of the contact-breaking process differs from the widespread "chewing gum" one, mentioned above, which assumes that a nanocontact has a form of a single narrow neck (constriction).

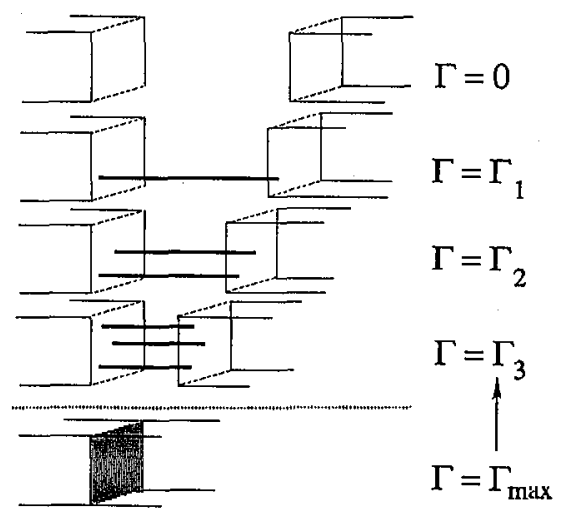

Fig. 2. Pictorial presentation of how the contact-breaking process is modelled. An initially closed (bottom) contact reveals fewer and fewer conduction paths as it is being open. 
We have carried out numerical studies of conductances of metallic contacts within the framework of the "semi-realistic" $s-d$ model with intraatomic hybridization described in Sec. 4. In contrast to the earlier studies (cf. Ref. [8] however, now the real-space representation is used and homogeneous metals are considered, i.e. the nanocontact (regarded as a quasiballistic conductor) is sandwiched between semi-infinite ideal lead wires (LW) made of the same metal. In the course of calculations the contact-breaking process has been mimicked by assuming that in last stages of the contact-breaking there are only a few conduction paths left, which are distributed at random over the cross-section area, and stretch across the nanocontact. The number of conduction paths is supposed to correspond to the breaking process duration (time). Technically, the conduction paths have been generated by putting additional huge on-site potentials everywhere within the nanocontact except on the paths. Lengths of the generated junctions (equal to the paths length) have been changed from 1 to 10 atomic spacings, and all the lengths have been assumed to occur with the same probability. The calculations presented below correspond to the cross-section equal to $7 \times 7$ (in lattice constant units), and 2000 random distributions for each number of the conduction channels. It is noteworthy that the present method differs from that of Ref. [17] in the following respect: (i) it applies to three dimensional systems and includes ferromagnets as well as paramagnets, (ii) it changes the number of conduction channels in a very natural way described above (keeping the Fermi energy fixed), and finally (iii) it introduces disorder quite practically in order to block some conduction paths during the contact breaking process. Figures $3 \mathrm{a}$ and $\mathrm{b}$ present cumulative histograms for
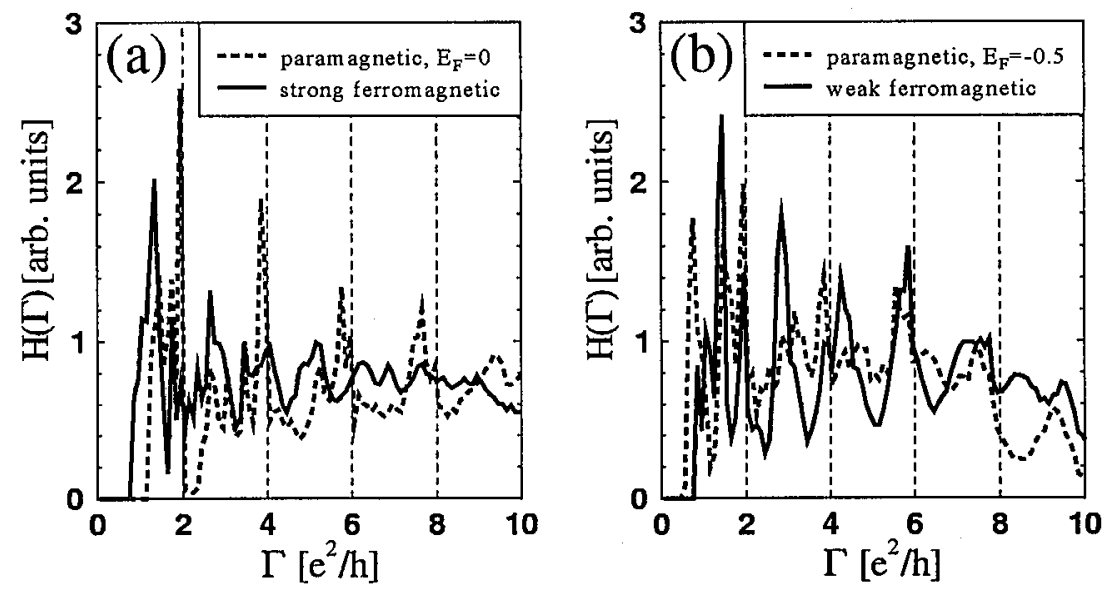

Fig. 3. Cumulative histograms of conductance. In the paramagnetic case, each histogram has got well-defined peaks. The peaks are still pronounced in the case of the weak ferromagnet (b). The positions of the main peaks can be fairly accurate brought to $2,4,6$, and $8 e^{2} / h$ if serial resistances of the order $200-300 \Omega$ are included. In contrast, for the strong ferromagnet (a), the peaks are much smaller and there is no way to drive them, by means of a single resistance added in series, to the above-mentioned even-integer positions. 
two qualitatively different situations, the former concerns the strong ferromagnet with $E_{\mathrm{F}}=0$ when the majority spin band is nearly completely full-filled, whereas the latter, the week ferromagnet case with $E_{\mathrm{F}}=-0.5$, has both the sub-bands only partially filled.

It is easily seen that in the paramagnetic cases there always are well pronounced peaks located close to $2,4,6,8 e^{2} / h$. Similar peaks are still present in the ferromagnetic case shown in Fig. 3b. Incidentally, the positions of the peaks can be brought even closer to the above-mentioned integers if an additional serial resistance $R$ (of the order of 200-300 $\Omega$ ), responsible for the so-called backscattering is included [11, 17], by simply modifying $\Gamma$ as follows: $\Gamma_{\text {real }}^{-1}=\Gamma^{-1}-R$.

In contrast to what has just been stated, in the ferromagnetic case of Fig. 3a the structure of the cumulative histogram is much less pronounced and there is no way to bring its peaks to the integer positions using only one extra resistor added in series. Thus the present model seems to yield results roughly consistent with recent experiments on $\mathrm{Ni}$ and $\mathrm{Fe}$. In particular for measurements performed in air, surface contaminations (oxidization) may lead easier to a featureless histogram for the former (strong ferromagnet), as observed in Ref. [13], than for the latter (weak ferromagnet) [10].

\section{Giant magnetoresistance and interlayer exchange coupling}

In this section we shall concentrate on CPP and CIP GMR in order to shed more light on the nature of these phenomena and their relation to interlayer exchange coupling $(J)$. The structures under consideration have a form of cubic trilayered (ferromagnet/paramagnet/ferromagnet) grains identical, for the

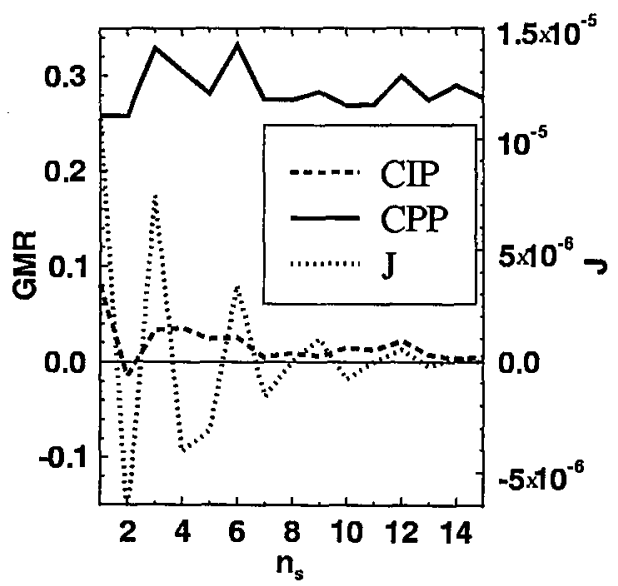

Fig. 4. GMR for the trilayer $n_{\mathrm{f}} / n_{\mathrm{s}} / n_{\mathrm{f}}$ with $n_{f}=3$ vs. spacer thickness $\left(n_{\mathrm{s}}\right)$. The CPP-GMR is much larger than the CIP-GMR, the former reveals predominantly short-period oscillations whereas the latter has got a quite pronounced long-period oscillation component. The dotted curve represents interlayer exchange coupling (r.h.s. scale). 
CIP and CPP calculations, in sizes and thicknesses of particular sub-layers. This makes it possible to compare in a reliable way the CIP and CPP results with each other.

In terms of the conductances $\Gamma$ defined in Eq. (7), the GMR is given by GMR $=\left(\Gamma_{\uparrow}^{\uparrow \uparrow}+\Gamma_{\downarrow}^{\uparrow \uparrow}\right) /\left(\Gamma_{\uparrow}^{\uparrow \downarrow}+\Gamma_{\downarrow}^{\uparrow \downarrow}\right)-1$, where the superscript $\uparrow \uparrow(\uparrow \downarrow)$ denotes the parallel (antiparallel) alignment of magnetic sub-layers.

In addition to the magnetoresistance we are interested in the mutual correlations between the GMR and the interlayer exchange coupling $(J)$, which has been determined from the configuration- and spin-dependent grand thermodynamic potentials (cf. [8])

$$
J=\Omega_{\uparrow}^{\uparrow \downarrow}+\Omega_{\downarrow}^{\uparrow \downarrow}-\Omega_{\uparrow}^{\uparrow \uparrow}-\Omega_{\downarrow}^{\uparrow \uparrow} .
$$

As can be seen from Fig. 4, plotted for the $n_{\mathfrak{f}} / n_{\mathrm{s}} / n_{\mathfrak{f}}$ trilayer with the ferromagnetic layer thickness $n_{\mathrm{f}}=3$, against the paramagnetic spacer thickness $n_{\mathrm{s}}$, the CPP-GMR is much greater than the CIP-GMR. Moreover, GMR in the CPP geometry reveals oscillatory behaviour with a short quasiperiod of about 3 monolayers (ML), whereas the CIP-GMR shows a long period of oscillations, too. The short period is also present in the interlayer exchange coupling $J$ represented by the dotted curve. Both the periods are consistent with asymptotic estimates (i.e. for $n_{\mathrm{s}} \longrightarrow \infty$ ), based on the extremal spanning vectors of the Fermi surfaces defined by the dispersion relation (Eq. (9)) with $E_{\mathrm{F}}=0$.

\section{Summary}

In the present paper the attention was focused on just two specific problems related with electrical conductance of small structures. It has been pointed out that in the case when dimensions of the system under consideration become small in comparison with some relevant characteristic length scales, the mesoscopic limit is reached. In this limit, under additional conditions of low temperature and no impurities, the electron transport may be ballistic. The ballistic conductance is expressed by remarkably simple formulas, containing only projections of the Fermi surface onto the plane perpendicular to the current direction. It has also been shown that it is possible to develop a more general formalism, in which certain requirements characteristic of the ballistic regime are relaxed. Such an approach, referred to as the quasiballistic one, is based on the Kubo formula and allows one to cope with nonideal systems too, e.g. with structural defects (used here to model the contact breaking process). As examples, two important problems have been discussed: (i) conductance through the contact breaking and (ii) magnetoconductance of trilayers for the CPP and CIP geometries. The former has led to a conclusion that electrical conductances are clearly spin-dependent and band structure details (band filling) seem to be decisive for the occurrence and the behaviour of discrete steps in the cumulative histograms. The latter, i.e. the GMR problem, shows that depending on the current orientation with respect to the layer, different periods of oscillations (determined by the Fermi surface calipers) can occur. Additionally it has been shown that the CPP-GMR is greater than the CIP-GMR and that their oscillations with the varying spacer thickness are correlated with the behaviour of the interlayer exchange coupling. 


\section{Acknowledgments}

This paper was supported by the Committee for Scientific Research grant No. 2P03B-118-14. I thank also the Poznań Supercomputing and Networking Center for the computing time.

\section{References}

[1] M. Johnson, Science 260, 12 April (1993).

[2] D.J. Monsma, J.C. Lodder, Th.J.A. Popma, B. Dieny, Phys. Rev. Lett. 74, 5260 (1995).

[3] Z. Wang, Y. Nakamura, J. Magn. Magn. Mater. 155, 161 (1996).

[4] J.M. Daughton, J. Magn. Magn. Mater. 192, 334 (1999).

[5] Yu.V. Sharvin, Zh. Eksp. Teor. Fiz. 48, 984 (1965); Sov Phys. JETP 21, 655 (1965).

[6] K.M. Schep, Ph.D. Thesis, University of Technology, Delft (NL) 1997.

[7] Y. Asano, A. Oguri, J. Inoue, S. Maekawa, J. Magn. Magn. Mater. 136, L18 (1994).

[8] S. Krompiewski, U. Krey, Europhys. Lett. 44, 661 (1998).

[9] S. Datta, Electronic Transport in Mesoscopic Systems, Eds. H. Ahmed, M. Pepper, A. Broers, Cambridge University Press, Cambridge 1995.

[10] F. Ott, Europhys. News 29 Jan./Feb., 13 (1998).

[11] B.J. van Wees, H. van Houten, C.W.J. Beenakker, J.G. Williamson, L.P. Kouvenhoven, D. van der Marel, C.T. Foxon, Phys. Rev. Lett. 60, 848 (1988).

[12] D.A. Wharam, T.J. Thornton, R. Newbury, M. Pepper, H. Ahmed, J.E.F. Frost, D.G. Hasko, D.C. Peacock, D.A. Ritchie, G.A.C. Jones, J. Phys. C 21, L 209 (1988).

[13] J.L. Costa-Krämer, Phys. Rev. B 55, R4875 (1997).

[14] F. Ott, S. Barberan, J.G. Lunney, J.M.D. Coey, P. Berthet, A.M. de Leon-Guevara, A. Revcolevschi, Phys. Rev. B 58, 4656 (1988).

[15] K. Hansen, E. Laegsgaard, I. Stensgaard, F. Besenbacher, Phys. Rev. B 56, 2208 (1997).

[16] H. Oshima, K. Miyano, Appl. Phys. Lett. 73, 2203 (1998).

[17] P. Garcia-Mochales, P.A. Serena, Phys. Rev. Lett 79, 2316 (1997). 